EPJ Web of Conferences 41, 02006 (2013)

DOI: $10.1051 /$ epjconf/20134102006

(C) Owned by the authors, published by EDP Sciences, 2013

\title{
Chirped Auger electron emission due to field-assisted post- collision interaction
}

\author{
B. Schütte ${ }^{1,3}$, S. Bauch ${ }^{2}$, U. Frühling ${ }^{1}$, M. Wieland ${ }^{1}$, M. Gensch ${ }^{4,5}$, E. Plönjes ${ }^{4}$, T. Gaumnitz ${ }^{1}$, \\ A. Azima ${ }^{1}$, M. Bonitz ${ }^{2}$, and M. Drescher ${ }^{1}$ \\ ${ }^{1}$ Universität Hamburg, Institut für Experimentalphysik, Luruper Chaussee 149, 22761 Hamburg, \\ Germany \\ ${ }^{2}$ Christian-Albrechts-Universität, Institut für Theoretische Physik und Astrophysik, Leibnizstr. 15, \\ 24098 Kiel, Germany \\ ${ }^{3}$ Max-Born-Institut, Max-Born-Str. 2A, 12489 Berlin, Germany \\ ${ }^{4}$ Deutsches Elektronen-Synchrotron DESY, Notkestr. 85, 22603 Hamburg, Germany \\ ${ }^{5}$ Helmholtz-Zentrum Dresden Rossendorf, Bautzner Landstr. 400, 01328 Dresden, Germany
}

\begin{abstract}
We have investigated the Auger decay in the temporal domain by applying a terahertz streaking light field. Xenon and krypton atoms were studied by implementing the free-electron laser in Hamburg (FLASH) as well as a source of high-order harmonic radiation combined with terahertz pulses from an optical rectification source. The observed linewidth asymmetries in the streaked spectra suggest a chirped Auger electron emission which is understood in terms of field-assisted post-collision interaction. The experimentally obtained results agree well with model calculations.
\end{abstract}

\section{Introduction}

In most cases, the Auger decay in atoms and molecules is studied in the spectral domain. A first direct measurement of the lifetime of Auger electrons from krypton atoms was provided in 2002, where a near-infrared (NIR) light field was applied to generate sidebands [1]. Here we present novel time-domain investigations of the Auger electron emission giving additional insight into its temporal dynamics. To this end, light-field streaking is applied which is capable of recording electron dynamics on an attosecond time scale [2]. For a transfer to processes occurring in the femtosecond (fs) range, terahertz $(\mathrm{THz})$ streaking fields are applied instead of the usual NIR fields. This new method of the THz-field-driven streak camera was successfully implemented for the extremeultraviolet (XUV) pulse characterization at the free-electron laser in Hamburg (FLASH) [3] as well as for a source of high-order harmonic generation (HHG) [4]. In this paper, we show evidence for an Auger electron chirp in the presence of a $\mathrm{THz}$ field. This effect is ascribed to the interaction of the Auger electron (AE), the photoelectron (PE) and the doubly charged ion, a process known as postcollision interaction (PCI) [5]. After providing theoretical considerations, we show experimental data and compare them to model calculations. unrestricted use, distribution, and reproduction in any medium, provided the original work is properly cited. 


\section{Theory}

PCI results in an energy transfer from the PE to its corresponding AE. It takes place when the AE emitted at a later time is faster than the PE. An AE with sufficient energy will overtake the PE at a certain distance from the ion and in this way be transferred from the doubly charged ionic potential curve to the singly charged ionic potential curve, since the charge is thereafter partly shielded by the PE. Without light field, the resulting spectrum is broadened (blue bold curves in Fig. 1). An applied $\mathrm{THz}$ field leads to an additional energy shift whose sign depends on the sign of the vector potential time derivative. The combination of these effects leads to a linewidth asymmetry and an energetic shift (cf. Fig. 1), which are a consequence of field-assisted post-collision interaction (FAPCI) [6].

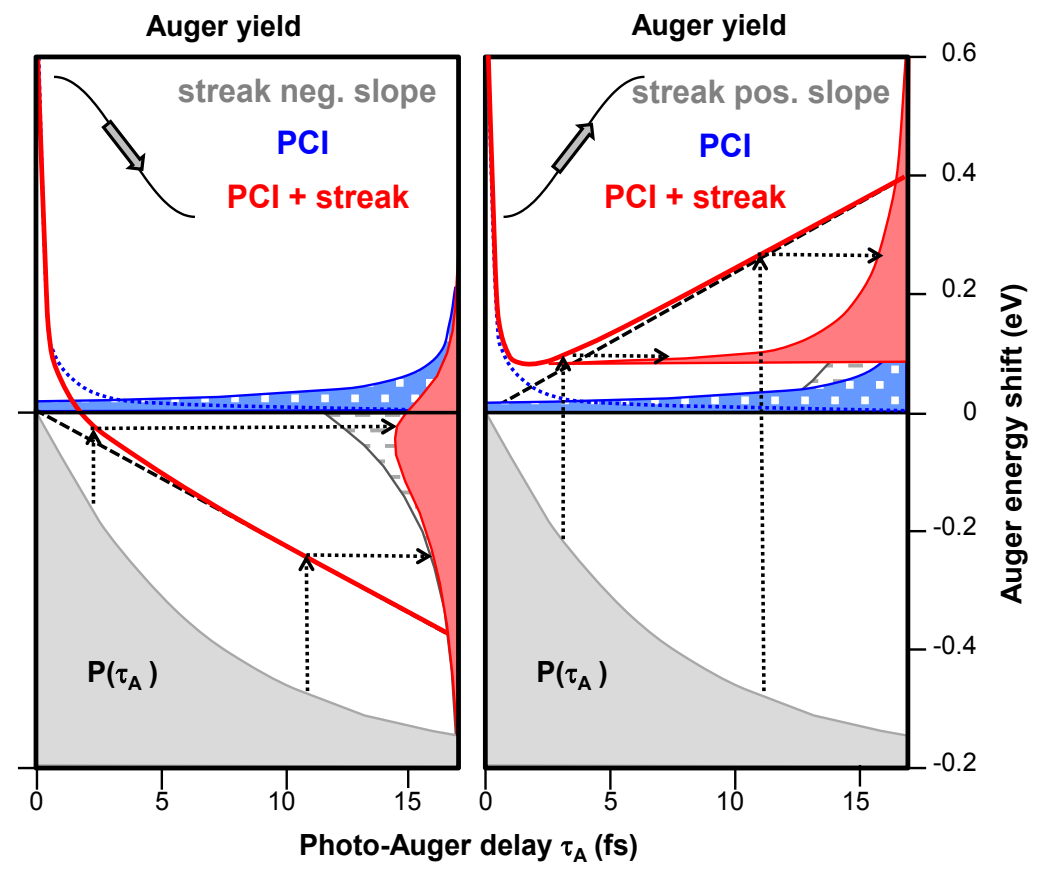

Fig. 1. Post-collision interaction in the presence of a $1 \mathrm{THz}$ light field at the positive (left side) and negative (right side) time derivative of the vector potential $A_{p}$. An Auger electron emitted at time $\tau_{A}$ experiences an energy gain due to PCI (dotted curve). The energy is additionally varied due to the changed phase of the $\mathrm{THz}$ field at the emission time (dashed curve). The combined effect of PCI and streaking leads to an energy distribution $\varepsilon\left(\tau_{A}, \mathrm{t}\right)$ (solid curve), which is narrower and energetically shifted at the negative slope with respect to the positive slope.

\section{Experiments}

The experiments were performed at two different light sources, namely at FLASH and at a HHG source, both exhibiting the same qualitative behaviour. Due to the better reproducibility of the HHG pulses and the higher flexibility, we will concentrate on results obtained with the laser-based system. Fig. 2 shows a streaking curve of Xe NOO Auger electrons and $4 d$ photoelectrons. Here the Xe atoms were ionized by $91 \mathrm{eV}$ XUV pulses in the presence of a THz field, and the emitted electrons were detected by a time-of-flight (TOF) spectrometer. All lines exhibit smaller widths at the first slope of the $\mathrm{THz}$ vector potential compared to the second slope (represented by the white arrows). Regarding the photoelectrons, this is an indication of a negatively chirped XUV pulse. Auger electrons, on the other hand, are not sensitive to the XUV energy and its change over time, i.e. a 
chirp. Here the linewidth asymmetry is ascribed to the process of FAPCI described above. Note that the slope of the kinetic energy shift has the opposite sign of the vector potential slope

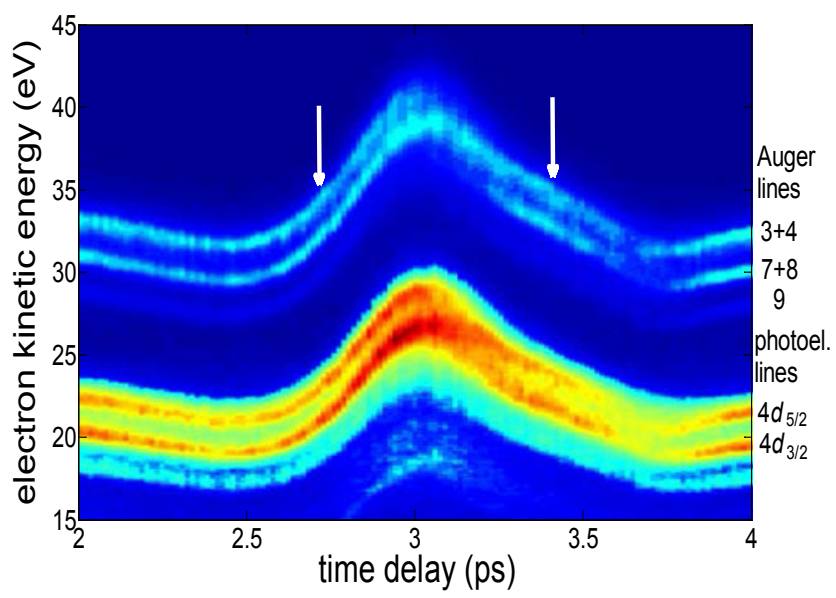

Fig. 2. Series of Xe $4 d$ photoelectron and $N O O$ Auger electron spectra at different time delays between the ionising XUV pulses and the THz streaking field. The linewidth asymmetry at the different slopes indicates a chirp. For the photoelectrons this is transferred from the XUV pulse, while for the Auger electrons the responsible mechanism is field-assisted post-collision interaction. Auger line numbers are taken from [7].

\section{Comparison and discussion}

For a quantitative comparison of experimental and theoretical data, extensive molecular dynamics calculations have been carried out that take into account angular distributions of the electrons. In addition, the XUV pulse duration of $20 \mathrm{fs}$ and the TOF resolution of $0.6 \mathrm{eV}$ were included. As a result, an excellent agreement was found between experimental and theoretical data in the $\mathrm{Xe}$ and $\mathrm{Kr}$ atoms under investigation, thus supporting evidence for the proposed model.

The obtained results demonstrate the capability of $\mathrm{THz}$ streaking to record electron dynamics on a fs time scale. In addition, there are practical implications of the current findings concerning XUV and $\mathrm{X}$-ray pulse duration measurements which typically rely on photoelectrons. For pulses of a few fs, however, the required $\mathrm{THz}$ field strengths are difficult to obtain. Instead, the linewidth asymmetry of Auger electrons could be exploited for XUV and X-ray pulse metrology in the future.

\section{References}

1. M. Drescher, M. Hentschel, R. Kienberger, M. Uiberacker, V. Yakovlev, A. Scrinzi, Th. Westerwalbesloh, U. Kleineberg, U. Heinzmann, F. Krausz, Nature 419, 803 (2002).

2. E. Goulielmakis, M. Uiberacker, R. Kienberger, A. Baltuska, V. Yakovlev, A. Scrinzi, Th. Westerwalbesloh, U. Kleineberg, U. Heinzmann, M. Drescher, F. Krausz, Science 305, 1267 (2004).

3. U. Frühling, M. Wieland, M. Gensch, T. Gebert, B. Schütte, M. Krikunova, R. Kalms, F. Budzyn, O. Grimm, J. Rossbach, E. Plönjes, M. Drescher, Nat. Photon. 3, 523 (2009).

4. B. Schütte, U. Frühling, M. Wieland, A. Azima, M. Drescher, Opt. Express 19, 18833 (2011).

5. R. B. Barker, H. W. Berry, Phys. Rev. 151, 14 (1966).

6. B. Schütte, S. Bauch, U. Frühling, M. Wieland, M. Gensch, E. Plönjes, T. Gaumnitz, A. Azima, M. Bonitz, M. Drescher, Phys. Rev. Lett. 108, 253003 (2012).

7. L. O. Werme, T. Bergmark, K. Siegbahn, Phys. Scr. 6, 141 (1972). 